\title{
Open-chain Peptides Obtained by Acidic Hydrolytic Cleavage of Cyclosporin A
}

\author{
F. Magni, C. Arcelloni, R. Paroni, I. Fermo, P. A. Bonini, M. Del Puppo, ${ }^{\text {a }}$ A. Manzocchia and M. \\ Galli Kienle ${ }^{\mathrm{a}}+$ \\ Scientific Institute H. S. Raffaele, Via Olgettina, 60, 20132 Milan, Italy

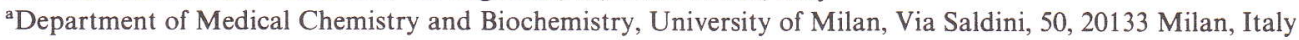

\begin{abstract}
Hydrolysis of cyclosporin A (CsA) was studied in order to clarify the still undefined point of attack of the acidic degradation. Among ether extractable and water-soluble products formed from $\mathrm{CsA}$ in $\mathrm{HCl}$, two open-chain peptides were isolated by high-performance liquid chromatography which were identified as the deca- and nonapeptides deriving from CsA through the hydrolytic cleavage of amino acid residue 11 and both residues 11 and 10 , respectively. Identification was carried out by fast atom bombardment tandem mass spectrometry.
\end{abstract}

\section{INTRODUCTION}

Cyclosporin A (CsA), the well-known immunosuppressant, ${ }^{1}$ is a cyclic undecapeptide. Metabolism of this peptide has been widely investigated and more than 30 compounds have been reported to be formed from CsA in vitro and/or in vivo. ${ }^{2}$ However, little attention has been given to the possible hydrolytic degradation of CsA, probably because inability of hydrolytic enzymes to degrade cyclic peptides has been reported ${ }^{3,4}$.

The hydrolytic cleavage of CsA by acids was described by the authors who first identified the primary structure of the cyclic undecapeptide CsA. ${ }^{5}$ Conditions utilized by these authors and by others ${ }^{6}$ were rather exhaustive, being selected to degrade the peptide structure completely. To the best of our knowledge nobody has ever isolated open-chain peptides longer than tetrapeptides from CsA degradation, so that the site of the first hydrolytic attack of the CsA molecule is still undefined.

In this paper we describe the isolation by highperformance liquid chromatography (HPLC) and identification by fast atom bombardment tandem mass spectrometry (FAB MS/MS) of two open-chain peptides among the products of CsA acid hydrolysis. The compounds, named decapeptide and nonapeptide, contain the $1-10$ and 1-9 residues of CsA, respectively, and show amino acid sequences unmodified with respect to that of CsA. This new finding and the formation of isocyclosporin A (isoCsA) by hydrolysis, as already reported by others, ${ }^{7-9}$ suggest the peptide bond between residues 1 and 11 of $\mathrm{CsA}$ to be the first one undergoing cleavage under acidic conditions. Identification of the two mentioned oligopeptides may be of relevance for the understanding of the possible degradative metabolism of CsA.

$\dagger$ Author to whom correspondence should be addressed.

\section{MATERIALS AND METHODS}

\section{Materials}

CsA was a generous gift of Dr G. Corbetta (Sandoz S.p.A., Milan, Italy). 3-Nitrobenzyl alcohol was from Aldrich Chimica (Milan, Italy). Deuterated solvents were from Merck (Darmstadt, Germany). Other reagents were of analytical grade. Solvents for highperformance liquid chromatography (HPLC) were used after being degassed and filtered using $0.45 \mu \mathrm{m}$ filters. Standard isoCsA was prepared and purified as described by Rüegger et al. ${ }^{5}$ by methansulphonic acid treatment of CsA, followed by silicic acid column chromatography. Purity of the compound was checked by thin-layer chromatography (TLC) using Silicagel 60 $\mathrm{F}_{254}$ plates (Merck, Darmstadt, Germany) eluted with chloroform-methanol $(98: 2, \mathrm{v} / \mathrm{v})$ containing $1 \%$ acetic acid $\left(R_{\mathrm{F}} 0.19\right)$ and by HPLC under the conditions described below (retention time $85 \mathrm{~min}$ ).

\section{Cyclosporin A hydrolysis}

Hydrolysis of CsA was carried out by mixing $1 \mu \mathrm{mol}$ of pure CsA (dried from an ethanolic solution) with $1 \mathrm{ml}$ of $3 \mathrm{M} \mathrm{HCl}$. The sample was heated at $60^{\circ} \mathrm{C}$ for $5 \mathrm{~h}$ and then dried under vacuum using a Savant centrifuge with mild heating $\left(45^{\circ} \mathrm{C}\right)$. The residue was dissolved with 1 $\mathrm{ml}$ of methanol-water $(1: 1, \mathrm{v} / \mathrm{v})$ and extracted twice with $1 \mathrm{ml}$ of diethyl ether. After evaporation of the organic solvent under nitrogen the residue was again dissolved in methanol-water. Both this solution and the aqueous phase were analysed by HPLC.

\section{HPLC analysis}

A System Gold liquid chromatograph (Beckman Instruments Inc., Palo Alto, California), with two dual-piston 


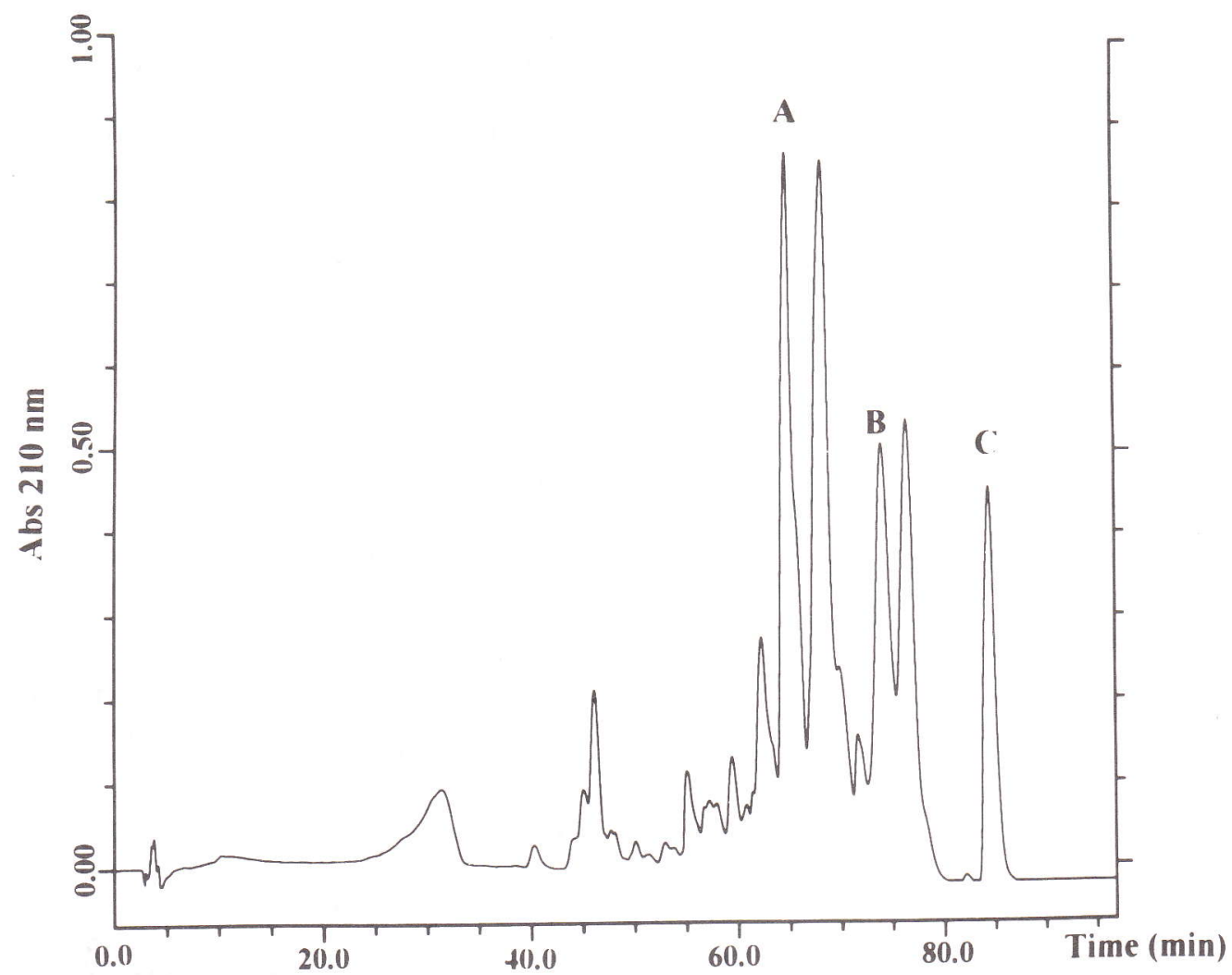

Figure 1. HPLC chromatogram of the aqueous phase obtained after hydrolysis of CsA. A, B and C correspond to compounds with retention times of 65,75 and $85 \mathrm{~min}$, respectively. CsA under these analytical conditions had a retention time of $105 \mathrm{~min}$ and was absent in the aqueous phase.

pumps (Mod. 126) and a variable double-beam ultraviolet (UV) detector (Mod. 166), was used. The apparatus was computer controlled (IBM, PS/50) and the System Gold software was used for the analysis of data. Peptide separation was carried out using a Beckman ODS Ultrasphere column $(150 \times 4.6 \mathrm{~mm}, 5 \mu \mathrm{m})$ eluted with $0.06 \%$ aqueous trifluoroacetic acid (TFA) (A) and $0.052 \%$ TFA in acetonitrile-water $(80: 20, \mathrm{v} / \mathrm{v})(\mathrm{B})$, performing a linear gradient of B from 0 to $100 \%$ in 105 min. ${ }^{10}$ The flow rate was $0.5 \mathrm{ml} \mathrm{min}{ }^{-1}$, and the UV detector was set at $210 \mathrm{~nm}$. Peaks of interest were collected and the solvent was evaporated under vacuum using a Savant centrifuge system.

\section{FAB mass spectrometric analysis}

In order to obtain information on the molecular weight of the unknown peptides spectra by FAB mass spectrometry were recorded using a Finnigan MAT95 double-focusing mass spectrometer equipped with a caesium gun, recording positive ions and operating at an ion accelerating voltage of $5 \mathrm{kV}$. The caesium ions were accelerated through $22 \mathrm{kV}$. The spectra were recorded from $\mathrm{m} / \mathrm{z} 100$ to $\mathrm{m} / \mathrm{z} 1500$ at $5-10 \mathrm{~s}$ per decade. The instrument was operated with a resolution of 1300 (10\% valley). 3 -Nitrobenzyl alcohol was used as matrix as previously reported by Paroni et al. ${ }^{11}$ for the analysis by FAB mass spectrometry of CsA and its metabolites. Dried fractions from HPLC were dissolved with methanol-water $(1: 1, \mathrm{v} / \mathrm{v})$ and $1 \mu \mathrm{l}$ aliquots were analysed. In order to exchange mobile hydrogens with deuterium the dried samples were dissolved in a 1:1 mixture of deuterated methanol-water and the FAB spectra were recorded using deuterated nitrobenzyl alcohol as the matrix.

To obtain additional structural information, collision-induced dissociation (CID) spectra of the protonated molecular ion $[\mathrm{M}+\mathrm{H}]^{+}$of the peptides were also recorded by introducing helium as collision gas into the first field-free region. The collision gas pressure in the collision cell was adjusted to attenuate the primary ion beam by $70 \%$. The CID spectra were obtained using the field-controlled $B / E$ linked scan in profile mode at $10 \mathrm{~s}$ per decade.

\section{Nuclear magnetic resonance analysis}

A Bruker AM-500 (Bruker Instruments, Silberpreiten, Germany) operating at $500 \mathrm{MHz}$ was used. Tetramethylsilane as the chemical shift reference was set at 0 ppm. Spectra of the decapeptide and nonapeptide were registered in deuterated water.

\section{RESULTS AND DISCUSSION}

HPLC analysis of the water-soluble compounds obtained by CsA hydrolysis under conditions generally used for the detection of oligopeptides ${ }^{10}$ showed the presence of relevant peaks probably originating from compounds with rather high molecular weight and/or low polarity, at retention times ranging from 65 to 90 
A

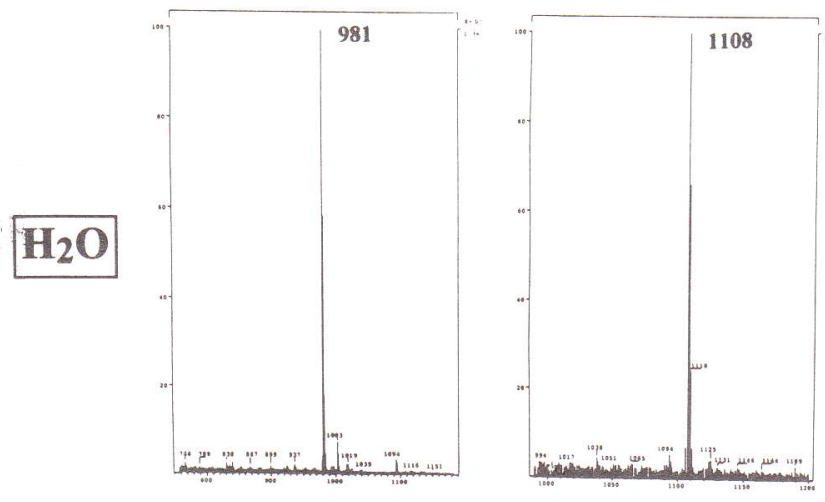

B
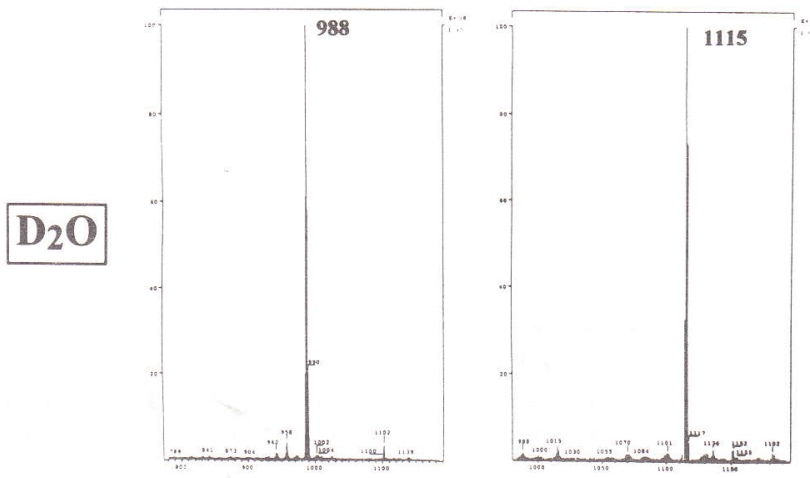

Figure 2. Mass spectra of the compounds with retention time 65 min and 75 min before (A) and after (B) treatment with deuterated water-methanol $(1: 1, \mathrm{v} / \mathrm{v})$. min, shorter than that of CsA (105 min). Peak area attributable to these compounds in the HPLC chromatogram corresponded to $37 \%$ of the total area, the remaining area being associated with peaks with shorter retention times (Fig. 1). Assuming for all compounds an unchanged molar absorption with respect to that of CsA, these compounds represented about $30 \%$ of the hydrolysed CsA. Qualitative GC/MS analysis under conditions described for the detection of amino acids ${ }^{12}$ suggested that part of CsA (10-15\%) had been hydrolysed completely; as described by others who used stronger hydrolytic conditions all amino acids that constitute the CsA chain were identified, excluding MeBmt. ${ }^{13}$

Compounds giving rise to the HPLC peaks shown in Fig. 1 were collected and taken to dryness. Analysis by FAB mass spectrometry of those with retention times of 65 and $75 \mathrm{~min}$ gave rise to spectra (Fig. 2A) with $[\mathrm{M}+\mathrm{H}]^{+}$ions at $m / z 981$ and 1108 , suggesting that they might derive from CsA by hydrolytic cleavage of the $\mathrm{MeBmt}{ }^{1}-\mathrm{MeVal}^{11}$ peptide bond followed by the loss of the $\mathrm{MeVal}^{11}$ or of both $\mathrm{MeVal}^{11}$ and MeLeu, ${ }^{10}$ respectively. The compound at retention time $85 \mathrm{~min}$ behaved as IsoCsA prepared according to Rüegger et $a l .{ }^{5}$ both by TLC and HPLC, and its FAB mass spectrum showed the $\left[\mathrm{M}+\mathrm{H}^{+}\right.$ion at $m / z$ 1203. The structure of compounds giving rise to peaks at retention times of 69 and $79 \mathrm{~min}$ is still under investigation.

The presence of ions at $m / z 1108$ and 981 in the FAB mass spectra recorded for the crude residue of the aqueous phase before HPLC analysis ensured that acidic conditions used for the HPLC purification were

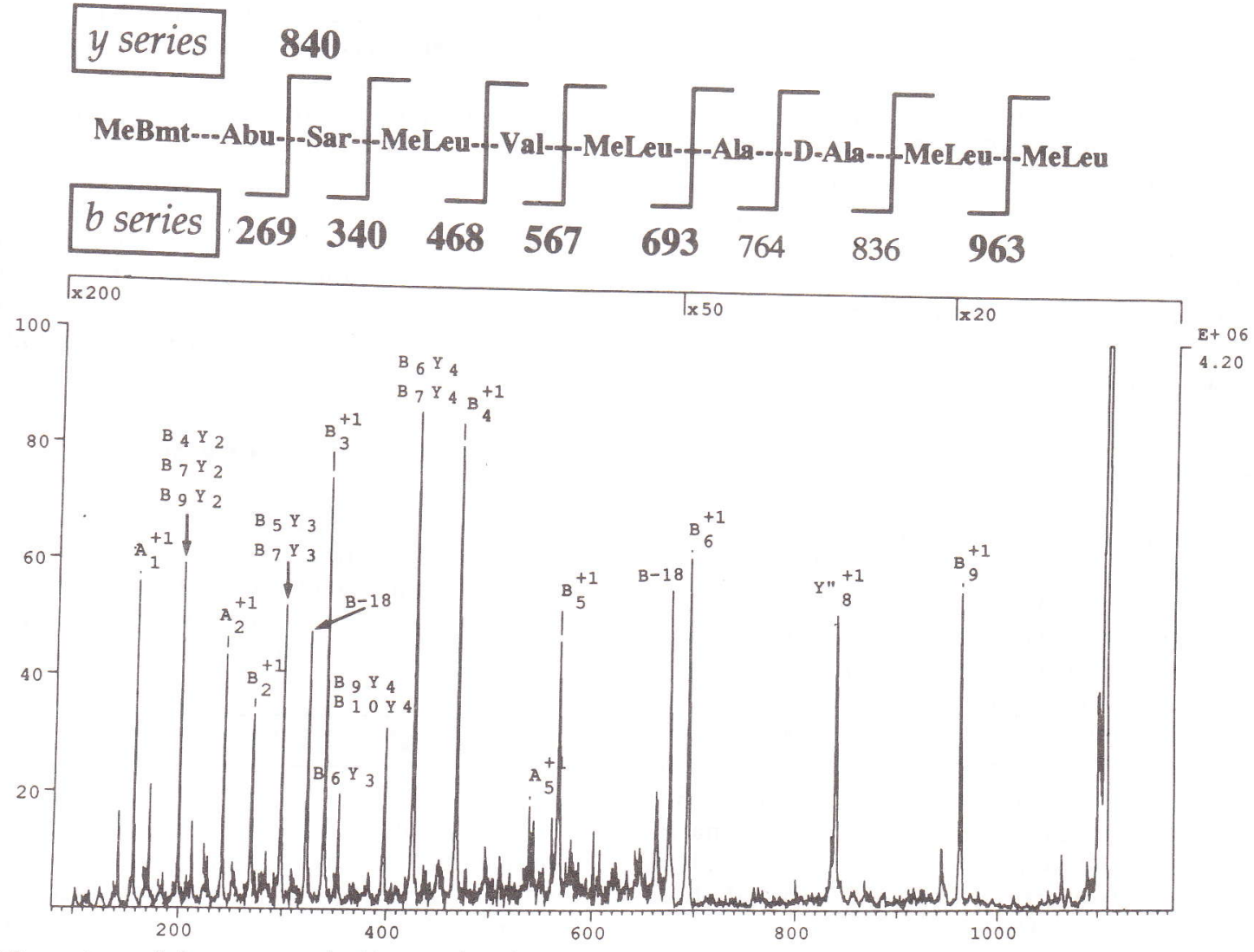

Figure 3. CID spectrum of the compound with retention time 75 min using helium as collision gas. The main observed fragmentation is reported on the spectrum. Among the indicated ions of the beries only those in bold characters are present in the CID. 

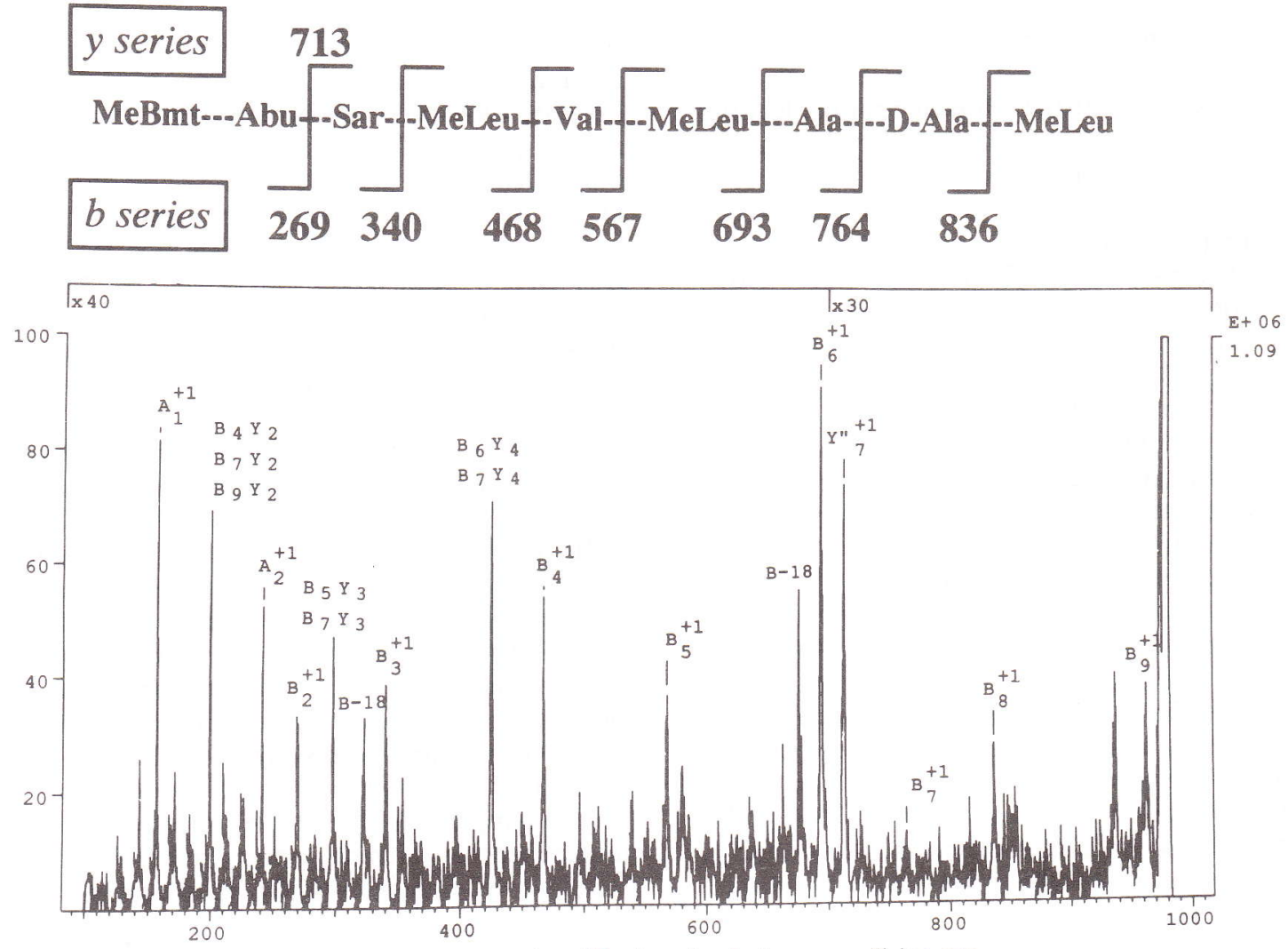

Figure 4. CID spectrum of the compound with retention time 65 min using helium as collision gas

not responsible for the formation of the peptides and that no further hydrolysis of these peptides occurred by drying the HPLC fraction containing TFA.

When spectra of the purified compounds were recorded after exchange of hydrogens with deuterium, the $[\mathrm{M}+\mathrm{H}]^{+}$signal was shifted by 7 mass units for both compounds (Fig. 2B) instead of the 8 units that would be expected on the basis of six $\mathrm{NH}$ and one $\mathrm{OH}$ groups in the hypothesized structures plus the protonating hydrogen. This may derive from a rapid exchange of either the protonating deuterium or of the deuterium of the hydroxy group with protium ions within the mass

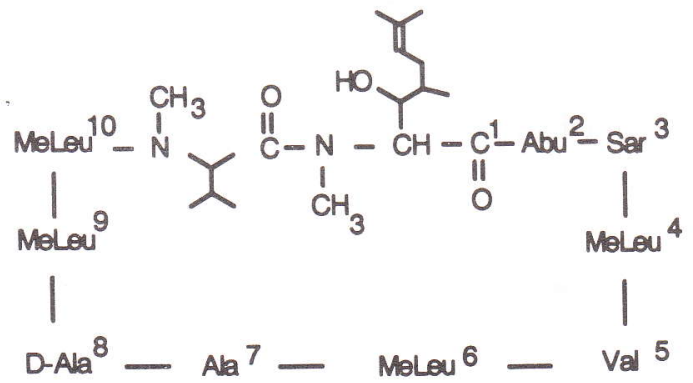

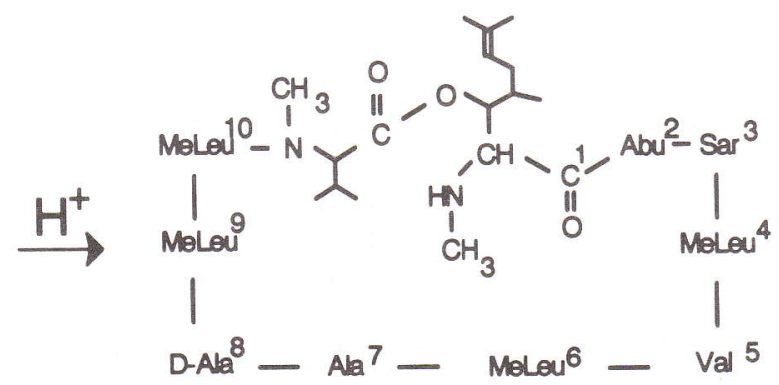

\section{CsA}

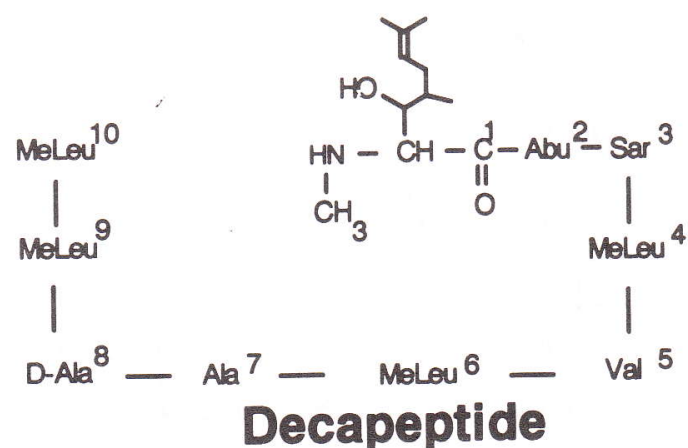

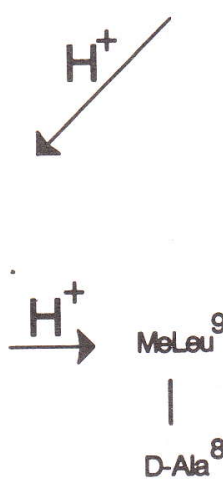

IsoCsA 
spectrometer. ${ }^{14}$ However, we cannot exclude the possibility that either before or during the ionization process isomerization may occur at the $\mathrm{MeBmt}$ residue, leading to a tetrahydrofuran ring ${ }^{13}$ with consequent loss of the mobile hydrogen of the hydroxy group of MeBmt.

Figure 3 shows the CID spectrum of the $[\mathrm{M}+\mathrm{H}]^{+}$ ion of the decapeptide. Major ions observed in the spectrum at $m / z 269,340,468,567,693$ and 963 corresponded to those expected for the $b$ series of the hypothetical decapeptide. For the $y$ series only the intense ion at $m / z 840$, corresponding to the $y_{8}^{\prime \prime}$, was noted. Identification of other relevant ions deriving from internal fragmentation of primary daughter ions is also reported in Fig. 3. Due to the repetitive residues in the primary structure of the peptide, these ions may occur by fragmentation of more than one daughter ion. In the CID spectrum of the nonapeptide reported in Fig. 4 a similar sequence of the ions was identified with a shift of 127 mass units for the ion at $m / z 840$ in the decapeptide CID spectrum, to $m / z$ 713. The absence of ion at $m / z 963$ in the latter spectrum suggested that a terminal MeLeu was missing in respect to the decapeptide. Other ions identified in the spectrum of decapeptide are also shown in Fig. 4. Mass values of ions of the b series present in the FAB tandem mass spectra deriving from fragmentation of the protonated molecular weight of the two peptides ensured for both compounds an unmodified primary structure with respect to that of the $\mathrm{MeBmt}^{1}-\mathrm{MeLeu}^{10}$ portion of CsA.

Due to the water solubility of the two compounds ${ }^{1} \mathrm{H}$-nuclear magnetic resonance spectra were recorded in deuterated water. Unfortunately no clear signals were obtained, probably due to the presence of various conformations of the peptides as observed for CsA by Ko and Davit. ${ }^{15}$

When this work had been already started, three papers on the treatment of CsA under mild conditions with aqueous $\mathrm{HCl}$ were published. ${ }^{7-9}$ However, formation of open-chain peptides was not mentioned. Under their hydrolytic conditions the authors of these papers observed only the isomerization of CsA to a compound that they identified by comparison of the HPLC retention time as isoCsA which was previously described by Rüegger et al. ${ }^{5}$ to be formed by methanesulphonic acid via acyl transfer from the peptide nitrogen of $\mathrm{MeVal}^{11}$ to the hydroxylic oxygen of $\mathrm{MeBmt}^{1}$ (Scheme 1). This compound was also formed under our hydrolytic conditions and was evidenced by HPLC and TLC analysis of the organic extracts, both showing in addition to residual cyclosporin (12-15\%) one peak representing $22 \%$ of CsA used as the substrate (retention time 85 min, Fig. 1, and $R_{\mathrm{F}} 0.17$, respectively). On the other hand Oliyai and Stella ${ }^{9}$ have reported that degradation of isoCsA occurs under rather mild acidic conditions and suggested the possibility that an open-chain peptide may derive from isoCsA degradation. The reason for the lack of identification by these authors of the peptides reported here may be the different substrate concentration and different conditions used for HPLC analysis.

Formation of the open-chain peptides described here by CsA hydrolysis suggests that acidic attack of the cyclic peptide chain occurs either at the $1-11,10-11$ or 9-10 peptide bond followed by elimination of the $\mathrm{MeVal}^{11}$ or both $\mathrm{MeVal}^{11}$ and MeLeu ${ }^{10}$ residues. Apparently no open-chain undecapeptide has been identified among the compounds formed by acid hydrolysis. Nevertheless isoCsA, whose formation implies the cleavage of the 1-11 peptide bond, was found in the reaction medium, suggesting that cleavage between the 1 and 11 residues might occur first (Scheme 1) and that isoCsA might be the stable form of the open-chain undecapeptide formed under mild acidic conditions (Scheme 1). Considering the amino acidic sequence of CsA we may suggest that the nona- and decapeptides accumulate owing to a lower rate of hydrolysis of $N$ methylated amino acids (MeVal and MeLeu), compared to those non-methylated (D-Ala and Ala) which follow in the sequence, and once eliminated would give rise to octa- and eptapeptides. This hypothesis seems to be validated by the behaviour of nona- and decapeptides to the collision with helium atoms. In fact in the CID spectra of both compounds ions deriving from Ala and D-Ala degradation are negligible or even absent.

In conclusion, results reported here suggest that the site of the first hydrolytic attack of the CsA molecule may be the 1-11 peptide bond. More importantly the possibility of obtaining from CsA aqueous soluble peptides which may possess biological activity is demonstrated.

\section{Acknowledgements}

This work was supported by the CNR Target Project 'Biotechnology and Bioinstrumentation'.

\section{REFERENCES}

1. B. D. Kahan, Transplant. Proc. XVII, 5 (1985).

2. B. D. Kahan, L. M. Shaw, D. Holt, J. Grevel and A. Johnston, Clin. Chem. 36, 1510 (1990).

3. H. L. Sham, C. A. Rempel, H. Stein and J. Cohen, J. Chem. Soc. Chem. Commun. 666 (1990).

4. R. F. Nutt, C. D. Colton, D. F. Veber, E. L. Slater and R. Saperstein, Klin. Wochenschr. 64, 71 (1986)

5. A. Rüegrer, M. Kuhn, H. Lichti, H. R. Loosli, R. Huguenin, C Quiquer and A. Wartburg, Helv. Chim. Acta 59, 1075 (1976).

6. N. R. Hartman, L. A. Trimble, J. C. Vederas and I. Jardine, Biochem. Biophys. Res. Commun. 133, 964 (1985).

7. G. J. Friis and H. Bungaard, Int. J. Pharm. 82, 79 (1992).
8. H. Bungaard and G. J. Friis, Int. J. Pharm. 82, 85 (1992).

9. R. Oliyai and V. J. Stella, Pharm. Res. 9, 617 (1992).

10. A. Yu. Rudensky, P. P. Hurlburt, S. Hong, A. Barlow and C. A. Janeway Jr, Nature 353, 622 (1991).

11. R. Paroni, I. Fermo, L. lavarone, M. Del Puppo, L. De Angelis and M. Galli Kienle, Pharmacol. Res. 28, 73 (1993)

12. G. L. Loy, A. N. Quick Jr, C. C. Teng, W. W. Hay Jr and P. V. Fennessey, Anal. Biochem. 185, 1 (1990).

13. N. R. Hartman and I. Jardine, Biomed. Mass Spectrom. 13 361 (1986)

14. J. A. McCloskey, in Methods in Enzymology, ed. by J. A McCloskey, p. 329. Academic Press, New York (1992)

15. S. Y. Ko and C. Davit, Int. J. Protein Res. 40, 380 (1992). 